\title{
Pyrimidine biosynthesis: the Achilles' heel of $T$. gondii?
}

De novo pyrimidine biosynthesis appears to be integral to the intracellular survival and virulence of Toxoplasma gondii and, therefore, may be the key to developing effective drugs and vaccines against this pathogen, report researchers from the US. ${ }^{1}$

They created two uracil auxotroph $T$. gondii strains (cps1-1 and cps2-1). These strains were genetically engineered to have no detectable carbamoyl phosphate synthetase II (CPSII) activity; CPSII is the first enzyme involved in the de novo pyrimidine biosynthesis pathway. Tachyzoites of both strains were found to be completely avirulent in immune-competent BALB/C mice and in gko mice that lack interferon- $\gamma$, a key cytokine involved in the mammalian immune response against $T$. gondii. Wild-type $T$. gondii (strain $\mathrm{RH}$ ) was lethal in both BALB/c and interferon- $\gamma$-deficient mice.

\section{Potential vaccine?}

Avirulent cps1-1 tachyzoites also induced a protective immune response in BALB/C mice. The researchers demonstrated that a single administration of $>10^{4}$ cps1-1 tachyzoites protected BALB/c mice against infection from a lethal dose of wild-type $T$. gondii.

These findings suggest that 'the obligate intracellular T. gondii parasite may have evolved to have a strict reliance on its own de novo pyrimidine biosynthesis pathway in vivo', and this adaptation 'may have arisen as a consequence of the apparent low availability of pyrimidines in animal tissues', comment the researchers.

They conclude that the de novo pyrimidine biosynthesis pathway in $T$. gondii could be a novel target for the design of antiparasitic drugs, but more importantly, avirulent uracil auxotroph strains 'may offer even greater promise as a strategy for vaccine development'.

In an accompanying commentary, Dr David Sibley from the Washington University School of Medicine, St Louis, US, agrees that these findings 'have implications for designing drugs to combat parasitic infections, and may offer a new vaccination approach'. ${ }^{2}$ He explains that parasitic and mammalian CPSII enzymes are inherently different and, therefore, 'it may be possible to design specific inhibitors of this enzyme that selectively disrupt pyrimidine synthesis in T. gondii but not in its hosts'. Furthermore, Dr Sibley says that the development of uracil auxotrophs may allow effective and reliable vaccination of livestock and this in turn may lead to fewer human infections.

\footnotetext{
1. Fox BA, et al. De novo pyrimidine biosynthesis is required for virulence of Toxoplasma gondii. Nature 415: 926-929, 21 Feb 2002.

2. Sibley LD. No more free lunch. Nature 415: 843-844, 21 Feb 2002.
} 